\title{
MEAN VALUES OF SUBSOLUTIONS OF ELLIPTIC AND PARABOLIC EQUATIONS
}

BY

WILLIAM P. ZIEMER ${ }^{1}$

\begin{abstract}
Integral averages of weak subsolutions (and supersolutions) in $R^{n}$ of quasilinear elliptic and parabolic equations are investigated. The important feature is that these integral averages are defined in terms of measures that reflect interesting geometric phenomena. Harnack type inequalities are established in terms of these integral averages.
\end{abstract}

1. Introduction. In the theory of regularity of weak solutions that arises in partial differential equations and the calculus of variations, the analysis of the set of Lebesgue points frequently plays an important role. A point $x_{0} \in R^{n}$ is said to be a Lebesgue point of a function $u$ if there is a number $l=l\left(x_{0}\right)$ such that

$$
\lim _{r \downarrow 0} r^{-n} \int_{B\left(x_{0}, r\right)}\left|u(y)-l\left(x_{0}\right)\right| d y=0 .
$$

Here $B\left(x_{0}, r\right)$ denotes the open ball of radius $r$ centered at $x_{0}$. If $u$ is a weak solution of a partial differential equation, certain regularity properties of $u$ often hold at its Lebesgue points. In many instances it is necessary to investigate the Lebesgue set and the associated integral averages of weak subsolutions and supersolutions. Perhaps the best known results in this connection are those that pertain to subharmonic functions. These results state that if $u$ is subharmonic in $R^{n}$, then $u$ has the following sub-mean-value properties:

$$
\begin{aligned}
& u\left(x_{0}\right) \leqslant\left(\alpha(n) r^{n}\right)^{-1} \int_{B\left(x_{0}, r\right)} u(y) d y, \\
& u\left(x_{0}\right) \leqslant\left(n \alpha(n) r^{n}\right)^{-1} \int_{\partial B\left(x_{0}, r\right)} u(y) d \sigma(y),
\end{aligned}
$$

where $\alpha(n)$ denotes the volume of the unit ball in $R^{n}$. Other results in this direction are the well-known Harnack inequalities for weak subsolutions and supersolutions of elliptic and parabolic equations [M, T1, T2].

In this paper we investigate integral averages of weak subsolutions of elliptic and parabolic quasilinear equations. This work was motivated by $[\mathbf{Z 1}]$ in which the

Received by the editors August 31, 1982.

1980 Mathematics Subject Classification. Primary 35J15, 35K65.

Key words and phrases. Lebesgue points, Harnack inequalities, Moser iteration.

${ }^{1}$ Research supported in part by the Mathematics Research Center, University of Wisconsin, contract No. DAAG29-80-C-0041 and by a grant from the National Science Foundation. 
analysis of Lebesgue points of weak subsolutions of parabolic equations played a vital role in determining the regularity properties of weak solutions of a wide class of degenerate parabolic equations.

In $\$ 4$ we consider weak subsolutions of equations of the form

$$
\operatorname{div} A\left(x, u, u_{x}\right)=B\left(x, u, u_{x}\right),
$$

where $A$ and $B$ are measurable functions subject to certain structural inequalities; see (12) below. Because of this structure, it is natural to require that weak subsolutions of (2) lie in the Sobolev space $W^{1, p}$. Our results for weak subsolutions $u$ of (2) are somewhat analogous to (1) in that we relate sup $u$ in $B\left(x_{0}, r\right)$ to the integral average of $u$ over an $(n-k)$-sphere of radius $r$ and center $x_{0}$ where $1 \leqslant k<p$. This is a special case of the main result which allows consideration of integral averages of subsolutions that are defined in terms of certain nonnegative measures (Theorem 4.6). Of course, similar results are valid for weak supersolutions. In $\$ 3$ we consider $u \in W^{1, p}$ without assuming that $u$ is a subsolution of (2) and show that the integral averages, that are defined relative to certain measures, converge everywhere to $u$ except possibly on a set whose dimension is $n-p$.

In $\$ 5$ we consider weak subsolutions of parabolic equations of the form

$$
u_{t}=\operatorname{div} A\left(x, t, u, u_{x}\right)+B\left(x, t, u, u_{x}\right) .
$$

We establish results which are analogous to those obtained in the elliptic case. In particular we show that the supremum of a weak subsolution of (3) in a cylinder of radius $r$ in $R^{n+1}$ can be estimated by its integral average over the lateral boundary of a suitable subcylinder plus an error term that tends to 0 as $r \rightarrow 0$. Other results of a similar nature can be obtained in which the lateral boundary of the subcylinder can be replaced by other geometric objects of dimension $n$.

The author would like to thank Michael Crandall for several interesting and helpful discussions during the preparation of this paper.

2. Notation and preliminaries. Points in Euclidean $n$-space, $R^{n}$, will be denoted by $x=\left(x_{1}, x_{2}, \ldots, x_{n}\right)$. In the case of a context for parabolic equations, points in $R^{n+1}$ will generally be denoted by $z=(x, t)$ where $x \in R^{n}$ and $t \in R^{1}$. We will denote $k$-dimensional Hausdorff measure by $H^{k}$. Thus, $H^{1}$ is linear measure and $H^{n}$ defined on subsets of $R^{n}$ is Lebesgue measure (cf. [F]). The Lebesgue measure of a set $E \subset R^{n}$ is denoted by $|E|$ and $f_{E} u(y) d \mu(y)$ will stand for the integral average $\mu(E)^{-1} \int_{E} u(y) d \mu(y)$, where $\mu$ is a nonnegative Radon measure with $\mu(E) \neq 0$. For $\Omega$ an open subset of $R^{n}, W^{1, p}(\Omega), \infty>p \geqslant 1$, will denote the Sobolev space of functions whose distributional first derivatives are functions that belong to the Lebesgue space $L^{p}(\Omega) . W_{0}^{1, p}(\Omega)$ will denote the closure in the Sobolev norm of smooth functions with supports contained in $\Omega$. Throughout, $p^{*}=n p /(n-p)$ will denote the Sobolev exponent, and $p^{\prime}=p /(p-1)$ for $1 \leqslant p<n$. Following common practice, the letter $C$ will denote a constant that may change from line to line in the same proof.

3. Mean values of Sobolev functions. Whenever $E \subset R^{n}$ we define, for $1 \leqslant p<n$,

$$
\gamma_{p}(E)=\inf \left\{\int|\nabla u|^{p}\right\}
$$


where the infimum is taken over all nonnegative functions $u \in W^{1, p}\left(R^{n}\right)$ for which $E \subset$ interior $\{x: u(x) \geqslant 1\}$. In the case $p \geqslant n$, the definition must be modified to require that the functions $u \in W^{1, p}\left(R^{n}\right)$ have supports contained in some fixed compact set. It is known (cf. [FZ]) that if $1<p \leqslant n$, then

$$
\gamma_{p}(E)=0 \text { implies } H^{n-p+\varepsilon}(E)=0 \text { for each } \varepsilon>0
$$

and

$$
H^{n-p}(E)<\infty \text { implies } \gamma_{p}(E)=0 .
$$

In the event that $p=1$, then $\gamma_{1}(E)=0$ if and only if $H^{n-1}(E)=0[\mathbf{F l}]$.

3.1. Definition. The Lebesgue set of degree $s$ for a Lebesgue measurable function $u$ is the set of all points $x \in R^{n}$ such that

$$
\lim _{r \downarrow 0} r^{-n} \int_{B(x, r)}|u(y)-u(x)|^{s} d y=0 .
$$

Here $B(x, r)$ denotes the open ball of radius $r$ and center $x$. The exceptional set of degree $s$ for $u$ is the complement in $R^{n}$ of the Lebesgue set of degree $s$ for $u$.

It follows from classical differentiation theory that in case $u \in L^{s}\left(R^{n}\right)$, the exceptional set of degree $s$ for $u$ has Lebesgue measure 0 . However, in case $u \in W^{1, p}\left(R^{n}\right)$ the exceptional set is considerably smaller. Indeed, we have the following (vide $[\mathbf{F Z}, \S 9]$ ):

3.2. TheOREM. For every $u \in W^{1 . p}\left(R^{n}\right), 1 \leqslant p<n$, there exists a function $v \in$ $W^{1 . p}\left(R^{n}\right)$ such that $u=v$ a.e. in $R^{n}$ and the exceptional set of degree $p^{*}$ for $v$ has $\gamma_{p}$ capacity 0 , where $p^{*}=n p /(n-p)$.

In particular, this results implies that the integral averages of $u$ converge at all points of $R^{n}$ except perhaps those that belong to a set of $\gamma_{p}$ capacity 0 ; that is,

$$
\lim _{r \downarrow 0} f_{B(x, r)} u(y) d y=u(x)
$$

for all $x \in R^{n}-E$, where $\gamma_{p}(E)=0$. The purpose of this section is to show that the integral average taken with respect to Lebesgue measure in (5) can be replaced by an average relative to a more general measure.

For this purpose we introduce the space of Bessel potentials $g_{1} * f, f \in L^{p}\left(R^{n}\right)$, $p>1$, where the Fourier transform of $g_{1}$ is given by $\hat{g}_{1}(x)=\left(1+4 \pi^{2}|x|^{2}\right)^{-1 / 2}$. The space of Bessel potentials $g_{1} * f, f \in L^{p}\left(R^{n}\right)$, is identical to $W^{1, p}\left(R^{n}\right), 1<p$ $<\infty$. The Bessel kernel $g_{1}$ is comparable to the Riesz kernel $R_{1}(x)=|x|^{1-n}$ in a neighborhood of $|x|=0$ and has exponential decrease as $|x| \rightarrow \infty$. Moreover, $g_{1}(x) \leqslant c|x|^{1-n}$ for some constant $C$.

3.3. Definition. For each real number $1 \leqslant \alpha<n$, let $M_{\alpha}^{+}$denote the space of nonnegative Radon measures $m$ on $R^{n}$ with compact support which, for some constant $M$, satisfy the growth condition $m\left(B_{r}\right) \leqslant M r^{n-\alpha}$ on open $n$-balls of radius $r$.

If $p>1$ and $m$ is a nonnegative Radon measure such that $\left\|g_{1} * m\right\|_{L^{p^{\prime}\left(R^{n}\right)}}<\infty$, where $p^{\prime}=p /(p-1)$, then clearly $m \in\left[W^{1, p}\left(R^{n}\right)\right]^{*}$, the dual of $W^{1, p}\left(R^{n}\right)$. To see this, let $u \in W^{1, p}\left(R^{n}\right)$ be written as $u=g_{1} * f, f \in L^{p}\left(R^{n}\right)$. Then, by changing the 
order of integration, we have

$$
\int_{R^{n}} u d m=\int g_{1} * f d m=\int g_{1} * m f d x \leqslant\left\|g_{1} * m\right\|_{p^{\prime}}\|f\|_{p}<\infty,
$$

and thus, $\int u d m$ defines a bounded linear form on $W^{1, p}\left(R^{n}\right)$. Again, by changing the order of integration, we have

$$
\int\left(g_{1} * m\right)^{p^{\prime}} d x=\int g_{1} *\left(g_{1} * m\right)^{1 / p-1} d m .
$$

Therefore, by appealing to Theorems 3.3 and 3.5 of [AM], it follows that if $p>1$, $1 \leqslant \alpha<p$ and $m \in M_{\alpha}^{+}$, then $m \in\left[W^{1, p}\left(R^{n}\right)\right]^{*}$. In case $p=1$, it follows from Theorem 4.7 of $[\mathbf{M Z}]$ that $m \in\left[W^{1,1}\left(R^{n}\right)\right]^{*}$ if $m \in M_{1}^{+}$. The main results of [M] and $[\mathbf{M Z}]$ thus yield the following

3.4. TheOREM. Let $\Omega \subset R^{n}$ be a bounded Lipschitz domain and suppose $1 \leqslant p<n$. Let $m$ be a nonnegative Radon measure supported on $\bar{\Omega}$ with $m \in M_{1}^{+}$if $p=1$ and $m \in M_{\alpha}^{+}$if $1 \leqslant \alpha<p$. There is a constant $C=C(\Omega, n, p, M)$ such that if $u \in$ $W^{1, p}(\Omega)$, then

$$
\left(\int_{\Omega}\left|u(y)-\int u d m\right|^{p^{*}} d y\right)^{1 / p^{*}} \leqslant C\left(\int_{\Omega}|\nabla u|^{p}\right)^{1 / p} .
$$

This result will be crucial in establishing (5) where Lebesgue measure in the integral average is replaced by $m \in M_{\alpha}^{+}$.

If $u \in W^{1, p}\left(R^{n}\right)$, define a measure $\gamma_{u}$ by

$$
\gamma_{u}(E)=\int_{E}|\nabla u|^{p} d x
$$

whenever $E$ is a measurable subset of $R^{n}$. Let

$$
P_{n-p}=R^{n} \cap\left\{x: \limsup _{r \downarrow 0} r^{p-n} \gamma_{u}[B(x, r)]>0\right\} .
$$

3.5. LemMA. If $u \in W^{1, p}\left(R^{n}\right), 1 \leqslant p<n$, then $H^{n-p}\left(P_{n-p}\right)=0$.

Proof. Let $A_{k}=R^{n} \cap\left\{x: \limsup _{r \downarrow 0} r^{p-n} \gamma_{u}[B(x, r)]>k^{-1}\right\}$ for each positive integer $k$. It follows from [F, §2.10.19] that there is a constant $C$ such that $H^{n-p}\left(A_{k}\right)$ $\leqslant C k \gamma_{\mu}\left(A_{k}\right)$. Note that $\gamma_{\mu}$ is absolutely continuous with respect to Lebesgue measure. Now $\gamma_{u}\left[A_{k}\right]<\infty, H^{n-p}\left(A_{k}\right)<\infty, H^{n}\left(A_{k}\right)=0, \gamma_{u}\left(A_{k}\right)=0$ and $H^{n-p}\left(A_{k}\right)=$ 0 . Hence $H^{n-p}\left(P_{n-p}\right)=0$ because $P_{n-p}=\cup A_{k}$.

Let $x_{0} \notin P_{n-p}$ and consider $B\left(x_{0}, 1\right)$. If $u \in W^{1, p}\left(R^{n}\right), 1 \leqslant p<n$, and if $m$ is a measure satisfying the hypotheses of Theorem 3.4 where we set $\Omega=B\left(x_{0}, 1\right)$, then

$$
\left(\int_{B\left(x_{0}, 1\right)}\left|u(y)-\int u d m\right|^{p^{*}} d y\right)^{1 / p^{*}} \leqslant C\left(\int_{B\left(x_{0}, 1\right)}|\nabla u|^{p}\right)^{1 / p} .
$$

Let $T_{r}: B\left(x_{0}, 1\right) \rightarrow B\left(x_{0}, r\right)$ be defined by $T_{r}\left(x_{0}+y\right)=x_{0}+r y$ where $y \in B(0,1)$. If we define $u_{r}=u \circ T_{r}$, then (6) implies that

$$
\left(\int_{B\left(x_{0}, 1\right)}\left|u_{r}(y)-\int u \circ T_{r} d m\right|^{p^{*}} d y\right)^{1 / p^{*}} \leqslant C\left(\int_{B\left(x_{0}, 1\right)}\left|\nabla u_{r}\right|^{p}\right)^{1 / p}
$$


which is the same as

$$
\left(r^{-n} \int_{B\left(x_{0}, r\right)}\left|u(y)-\int u \circ T_{r} d m\right|^{p^{*}} d y\right)^{1 / p^{*}} \leqslant C\left(r^{p-n} \int_{B\left(x_{0}, r\right)}|\nabla u|^{p}\right)^{1 / p} .
$$

Because $x_{0} \notin P_{n-p}$, the right side of (7) tends to 0 as $r \downarrow 0$. From Theorem 2.2 there is a set $E$ with $\gamma_{p}(E)=0$ such that

$$
\lim _{r \downarrow 0} r^{-n} \int_{B\left(x_{0}, r\right)}\left|u(y)-u\left(x_{0}\right)\right|^{p^{*}} d y=0
$$

whenever $x_{0} \notin E$. Note that (4) and Lemma 3.5 imply $\gamma_{p}\left(P_{n-p}\right)=0$. Now by setting $A=E \cup P_{n-p}$, the next result follows from (7) and (8).

3.6. TheOREM. Suppose $u \in W^{1, p}\left(R^{n}\right), 1 \leqslant p<n$. There is a set $A \subset R^{n}$ with $\gamma_{p}(A)=0$ such that if $x_{0} \notin A$ and $m$ is a nonnegative Radon measure supported on $\vec{B}\left(x_{0}, 1\right)$ with $m \in M_{1}^{+}$if $p=1$ and $m \in M_{\alpha}^{+}$if $1 \leqslant \alpha<p$, then

$$
\lim _{r \downarrow 0} \int u \circ T_{r} d m=u\left(x_{0}\right) .
$$

Of course, the most interesting measures to consider are those that possess some homogeneity properties. For example, if $m=H^{n-1} \mid \partial B\left(x_{0}, 1\right)$, then clearly $m \in M_{1}^{+}$ and

$$
\int u \circ T_{r} d m=f_{\partial B\left(x_{0}, r\right)} u(y) d H^{n-1}(y)
$$

Thus, we have

3.7. Corollary. If $u \in W^{1,1}\left(R^{n}\right)$ there is a set $A$ with $\gamma_{1}(A)=H^{n-1}(A)=0$ such that

$$
\lim _{r \downarrow 0} f_{\partial B\left(x_{0}, r\right)} r(y) d H^{n-1}(y)=u\left(x_{0}\right)
$$

whenever $x_{0} \notin A$.

A similar result could be obtained by taking $m=H^{n-1} \mid \pi^{n-1}\left(x_{0}\right)$ where $\pi^{n-1}\left(x_{0}\right)$ is an $(n-1)$-dimensional plane passing through $x_{0}$. In the event that $u \in W^{1, p}\left(R^{n}\right)$, $1<p<n$, let $m=H^{n-R} \mid S^{n-k}\left(x_{0}, 1\right)$ where $1 \leqslant k<p$ is an integer and $S^{n-k}\left(x_{0}, 1\right)$ is an $S^{n-k}$ sphere with radius 1 centered at $x_{0}$. Then we have

$$
\lim _{r \downarrow 0} f_{S^{n-k}\left(x_{0}, r\right)} u(y) d H^{n-k}(y)=u\left(x_{0}\right)
$$

for $\gamma_{p}$-a.e. $x_{0} \in R^{n}$. As in the case $p=1$, a similar result follows by taking $m=H^{n-k} \mid \pi^{n-k}\left(x_{0}\right)$.

4. Lebesgue points of subsolutions of elliptic equations. In this section we obtain results for weak subsolutions and supersolutions of quasilinear elliptic equations with measurable coefficients that are analogous to Theorem 3.6 and its corollaries. In the case of weak subsolutions we will show that (9) holds at all points $x_{0}$ and, more importantly, that the sup $u$ in $B\left(x_{0}, r\right)$ is bounded above by the left-hand side of (9) plus a term that tends to 0 as $r \downarrow 0$. 
The equations that are considered in this section are of the form

$$
\operatorname{div} A\left(x, u, u_{x}\right)=B\left(x, u, u_{x}\right)
$$

where $A$ and $B$ are, respectively, vector and scalar valued Baire functions defined on $\Omega \times R^{1} \times R^{n}$. Here $\Omega$ is an open subset of $R^{n}$. The functions $A$ and $B$ are required to satisfy the following structural inequalities:

$$
\begin{gathered}
|A(x, u, w)| \leqslant a_{0}|w|^{p-1}+a_{1}|u|^{p-1}+a_{2}, \\
|B(x, u, w)| \leqslant b_{0}|w|^{p}+b_{1}|w|^{p-1}+b_{2}|u|^{p-1}+b_{3}, \\
A(x, u, w) \cdot w \geqslant|w|^{p}-C_{1}|u|^{p}-C_{2} .
\end{gathered}
$$

We assume $1<p<n$ and $a_{0}, b_{0}$ are nonnegative constants. The results below are valid if the remaining coefficients are nonnegative measurable functions that are assumed to lie in appropriate $L^{q}(\Omega)$ spaces (vide [GZ]), but to minimize technical detail, we will assume that the coefficients are bounded by some constant $K>0$ :

$$
a_{i}(x) \leqslant K, \quad b_{i}(x) \leqslant K, \quad c_{i}(x) \leqslant K .
$$

A function $u \in W_{\text {loc }}^{1, p}(\Omega)$ is called a weak subsolution (supersolution) of (11) if

$$
\int_{\Omega} A\left(x, u, u_{x}\right) \cdot \nabla \phi+B\left(x, u, u_{x}\right) \phi \leqslant 0 \quad(\geqslant 0)
$$

for all bounded $\phi \geqslant 0, \phi \in W_{0}^{1, p}(\Omega)$.

The following result is due to Trudinger [T1] whose proof is based on the familiar Moser iteration method [Mo]. Therefore, a complete proof will not be given, but for the convenience of the reader, we will give an outline of the main steps.

Let $u \in W_{\text {loc }}^{1, p}(\Omega)$ be a weak subsolution of (11) that is bounded above by $L$ in $\Omega$. Choose $x_{0} \in \Omega$, and for each real number $k$, let $u_{k}=(u-k)^{\dagger}$ and define

$$
\begin{aligned}
\mu_{k}(r) & =\sup \left\{u_{k}(x): x \in B\left(x_{0}, r\right)\right\}, \\
\mu(r) & =\sup \left\{u(x): x \in B\left(x_{0}, r\right)\right\} .
\end{aligned}
$$

4.1. THEOREM. Let $u \in W_{\text {lox }}^{1, p}(\Omega), 1<p<n$, be a weak subsolution of (11) such that $0 \leqslant u(x) \leqslant L$ for each $x \in \Omega$. There is a constant $C$ depending only on $L, n, p$ and the structure (12) such that if $B\left(x_{0}, r\right) \subset \Omega$, then

$$
\left(f_{B\left(x_{0}, 7 r / 8\right)}|\mu(r)-u|^{\gamma}\right)^{1 / \gamma} \leqslant C[\mu(r)-\mu(r / 2)+a(r)],
$$

where $a(r)=r+K r+(K r)^{p /(p-1)}$ and $\gamma<n(p-1) /(n-p)$.

Proof. Let

$$
\phi=\eta^{p} e^{-b_{0} u}(\mu(r)+a(r)-u)^{\alpha},
$$

where $\eta \in C_{0}^{\infty}\left[B\left(x_{0}, r\right)\right]$ and $\alpha<0$. Then $\phi$ is a bounded, nonnegative test function that can be employed in (14). If we set $v=\mu(r)+a(r)-u$, the structure (12) and elementary estimates yield

$$
\int \eta^{p} v^{\alpha-1}|\nabla v|^{p} \leqslant C(\alpha) \int\left(\eta^{p}+|\nabla \eta|^{p}\right) v^{p+\alpha+1},
$$


where $C(\alpha)$ is a finite constant when $\alpha$ is bounded away from 0 and $1-p$. Let $w=v^{q}$ where $p q=p+\alpha-1$ and apply Sobolev's inequality to find that (17) yields

$$
\|\eta w\|_{\sigma p} \leqslant C(\alpha)\|(\eta+|\nabla \eta|) w\|_{p}
$$

where $\sigma=n /(n-p)$. For $0<s<t \leqslant r$, let $\eta \in C_{0}^{\infty}\left[B\left(x_{0}, t\right)\right]$ be such that $0 \leqslant \eta \leqslant$ $1, \eta \equiv 1$ on $B\left(x_{0}, s\right)$ and $|\nabla \eta| \leqslant 2(t-s)^{-1}$. Then (18) implies

$$
\|w\|_{\sigma p: s} \leqslant C(\alpha)(t-s)^{-p}\|w\|_{p ; t} .
$$

Let $r_{j}=r\left(2^{-1}+2^{-j-2}\right)$ for $j=0,1, \ldots$ and iterate inequality (19) to obtain for any $p_{0}>0$,

$$
\theta(-\infty, r / 2) \geqslant C \theta\left(-p_{0}, 3 r / 4\right)
$$

where

$$
\theta(p, r)=\left(f_{B\left(x_{0}, r\right)} v^{p}\right)^{1 / p}
$$

By performing a finite iteration of (19) and choosing the $r_{j}$ in a different way, we have

$$
\theta(\gamma, 7 r / 8) \leqslant C \theta\left(p_{0}, 3 r / 4\right)
$$

for any $p_{0}>0$ and $\gamma<n(p-1) /(n-p)$. Return now to the definition of the test function $\phi$ and notice that in case $\alpha=-1$, substitution of $\phi$ into (14) gives

$$
\int \eta^{p}|\nabla \log v|^{p} \leqslant C \int|\nabla \eta|^{p}
$$

whenever $\eta \in C_{0}^{\infty}\left[B\left(x_{0}, r\right)\right]$. Thus, from the John-Nirenberg lemma (cf. [GT, p. 158]), it follows that there exist constants $C$ and $p_{0}>0$ such that

$$
\int_{B\left(x_{0}, s\right)} v^{-p_{0}} \int_{B\left(x_{0}, s\right)} v^{p_{0}} \leqslant C S^{2 n}
$$

for $0<s \leqslant 3 r / 4$. That is,

$$
\theta\left(p_{0}, 3 r / 4\right) \leqslant \theta\left(-p_{0}, 3 r / 4\right) .
$$

Thus, (20)-(22) imply

$$
\theta(\gamma, 7 r / 8) \leqslant C \theta(-\infty, r / 2)
$$

or

$$
\begin{aligned}
\left(f_{B\left(x_{0}, 7 r / 8\right)}[\mu(r)+a(r)-u]^{\gamma}\right)^{1 / \gamma} & =\left(f_{B\left(x_{0}, 7 r / 8\right)} v^{\gamma}\right)^{1 / \gamma} \\
& \leqslant C \min _{B\left(x_{0}, r / 2\right)} v=C[\mu(r)-\mu(r / 2)+a(r)] .
\end{aligned}
$$

4.2. Remark. Note that the right-hand side of the inequality in Theorem 4.1 tends to 0 with $r$ and therefore

$$
\lim _{r \downarrow 0} f_{B\left(x_{0}, r\right)} u(y) d y=\lim _{r \downarrow 0} \mu(r)=\lim _{x \rightarrow x_{0}} \sup u(x) .
$$


This implies that every bounded weak subsolution of (11) is upper semicontinuous on $\Omega$ after redefinition on a set of measure 0 .

If $b_{0}=0$ in the structure (12), then the same conclusion will hold without assuming that $u$ is bounded on $\Omega$. In this case it is possible to show that a weak subsolution $u \in W_{\text {loc }}^{1, p}(\Omega)$ is locally bounded above on $\Omega$ [S]. On the other hand, $u$ is not necessarily bounded below and, therefore, the estimate in Theorem 3.1 may not hold. However, one can easily show that for each $k \in R^{1}$,

$$
\lim _{r \downarrow 0} f_{B\left(x_{0}, 7 r / 8\right)}\left|\mu_{k}(r)-u_{k}\right|^{\gamma}=0 .
$$

To see this, replace $u$ by $u_{k}$ and $\mu(r)$ by $\mu_{k}(r)$ in the definition of $\phi,(16)$, and observe that the proof of Theorem 3.1 yields

$$
\left(f_{B\left(x_{0}, 7 r / 8\right)}\left|\mu_{k}(r)-u_{k}\right|^{\gamma}\right)^{1 / \gamma} \leqslant C(k)\left[\mu_{k}(r)-\mu_{k}(r / 2)+a(r)\right] .
$$

Let $\lambda\left(x_{0}\right)=\lim _{r \downarrow 0} \mu(r)$ and choose $k<\lambda\left(x_{0}\right)$. Then

$$
\int_{B\left(x_{0}, 7 r / 8\right)} \mu_{k}(r)-u_{k} \geqslant\left(\lambda\left(x_{0}\right)-k\right)\left|B\left(x_{0}, 7 r / 8\right) \cap\{u<k\}\right|
$$

and, thus, it follows from (23) that

$$
\lim _{r \downarrow 0} r^{-n}\left|B\left(x_{0}, r\right) \cap\{u<k\}\right|=0 .
$$

Therefore, for each $\varepsilon>0$,

$$
\lim _{r \downarrow 0} r^{-n}\left|B\left(x_{0}, r\right) \cap\left\{x:\left|u(x)-\lambda\left(x_{0}\right)\right|>\varepsilon\right\}\right|=0 .
$$

This states that $u$ is approximately continuous at $x_{0}$ and its approximate limit at $x_{0}$ is $\lambda\left(x_{0}\right)$. However, every measurable function is approximately continuous almost everywhere and, therefore, every weak subsolution of (11) with $b_{0}=0$ in (12) is upper semicontinuous on $\Omega$ after redefinition on a set of measure 0 .

We will now show that (9) holds at all $x_{0} \in \Omega$ if $u$ is a subsolution of (11).

4.3. THEOREM. Let $u \in W_{\mathrm{loc}}^{1, p}(\Omega), 1<p<n$, be a weak subsolution of (11) such that $0 \leqslant u(x) \leqslant L$ for each $x \in \Omega$. For each $x_{0} \in \Omega$ with $B\left(x_{0}, r_{0}\right) \subset \Omega$ for some $r_{0}>0$, let $m \in M_{\alpha}^{+}, 1 \leqslant \alpha<p$, be a Radon measure supported on $\bar{B}\left(x_{0}, r_{0} / 2\right)$. There is a constant $C$ depending only on $M, L, n, p$ and the structure (12) such that

$$
\left(r^{-n} \int_{B\left(x_{0}, r\right)}\left|u(y)-\int u \circ T_{r} d m\right|^{p^{*}} d y\right)^{1 / p^{*}} \leqslant C[\mu(r)-\mu(r / 2)+a(r)]^{p-1 / p}
$$

for each $r \leqslant r_{0} / 2$ where $T_{r}: B\left(x_{0}, r_{0} / 2\right) \rightarrow B\left(x_{0}, r / 2\right)$ is defined by $T_{r}\left(x_{0}+y\right)=x_{0}$ $+r / r_{0} y$.

Proof. We proceed to obtain an estimate of $\int_{B\left(x_{0}, r\right)}|\nabla u|^{p}$ as in [GZ]. Let $\eta \in C_{0}^{\infty}\left[B\left(x_{0}, 7 r / 8\right)\right]$ be a cut-off function such that $\eta \equiv 1$ on $B\left(x_{0}, r / 2\right)$ and let 
$\phi=\eta^{p} e^{h_{0} u}$. Hence, by substituting $\phi$ into (14) and utilizing the structure (12), it follows that there is a constant $C$ such that

$$
\int \eta^{p}|\nabla u|^{p} \leqslant C\left[\int \eta^{p-1}|\nabla \eta|^{p-1}|\nabla \eta|+\int \eta^{p}+\int \eta^{p-1}|\nabla \eta|+\int \eta^{p}|\nabla u|^{p-1}\right] .
$$

As in the proof of Theorem 4.1, let $v=\mu(r)-u+a(r)$ and use (17) and Theorem 4.1 to obtain, for sufficiently small $\varepsilon>0$,

$$
\begin{aligned}
\int \eta^{p-1}|\nabla u|^{p-1}|\nabla \eta|= & \int\left(\eta^{p-1} v^{-(1+\varepsilon) / p^{\prime}}|\nabla u|^{p-1}\right)\left(v^{(1+\varepsilon) / p^{\prime}}|\nabla \eta|\right) \\
\leqslant & \left(\int \eta^{p} v^{-(1+\varepsilon)}|\nabla v|^{p}\right)^{p-1 / p}\left(\int v^{(p-1)(1+\varepsilon)}|\nabla \eta|^{p}\right)^{1 / p} \\
\leqslant & C\left(\int\left(\eta^{p}+|\nabla \eta|^{p}\right) v^{p-1-\varepsilon}\right)^{p-1 / p} \\
& \times\left([\mu(r)-\mu(r / 2)+a(r)]^{(p-1)(1+\varepsilon)} \cdot r^{n-p}\right)^{1 / p} \\
\leqslant & C\left([\mu(r)-\mu(r / 2)+a(r)]^{(p-1-\varepsilon)} r^{n-p}\right)^{p-1 / p} \\
& \times\left([\mu(r)-\mu(r / 2)-a(r)]^{(p-1)(1+\varepsilon)} r^{n-p}\right)^{1 / p} \\
\leqslant & C[\mu(r)-\mu(r / 2)+a(r)]^{p-1} r^{n-p} .
\end{aligned}
$$

Also, the last term in (25) can be written as

$$
\int \eta^{p}|\nabla u|^{p-1}=\int(\eta|\nabla u|)^{p-1} \eta \leqslant \frac{1}{2} \int \eta^{p}|\nabla u|^{p}+C \int \eta^{p}
$$

Hence, it follows from (25) and (26) that

$$
r^{p-n} \int_{B\left(x_{0}, r / 2\right)}|\nabla u|^{p} \leqslant C[\mu(r)-\mu(r / 2)+a(r)]^{p-1} .
$$

Now apply Theorem 3.4 as in the proof of (7) to establish the conclusion.

Because the right-hand side of (24) tends to 0 as $r \downarrow 0$, the following is immediate.

4.4. Corollary. If $u \in W_{\text {loc }}^{1, p}(\Omega)$ is a bounded subsolution of (11) on $\Omega$, then

$$
\lim _{r \downarrow 0} \int u \circ T_{r} d m=u\left(x_{0}\right)
$$

for each $x_{0} \in \Omega$.

4.5. REMARK. As in (10), if we let $m=H^{n-k} \mid S^{n-k}\left(x_{0}, r_{0} / 2\right)$, we have for each $x_{0} \in \Omega$,

$$
\lim _{r \downarrow 0} f_{S^{n-k}\left(x_{0}, r\right)} u(y) d H^{n-k}(y)=u\left(x_{0}\right)
$$

whenever $k$ is an integer such that $1 \leqslant k<p$. A similar result holds if $m$ is taken as the restriction of $H^{n-k}$ to an $(n-k)$-plane passing through $x_{0}$. 
Finally, we establish an inequality for weak subsolutions of (11) which is reminiscent of the weak Harnack inequalities proved in [T1].

4.6. TheOREM. Let $u \in W_{\mathrm{loc}}^{1, p}(\Omega), 1<p<n$, be a weak subsolution of (11) and assume that $0 \leqslant u \leqslant L$ on $\Omega$. For each $x_{0} \in \Omega$ with $B\left(x_{0}, r_{0}\right) \subset \Omega$ for some $r_{0}>0$, there is a nonnegative function $g(r)$ with $\int_{0} g(r)^{p^{\prime}} d r / r<\infty$ such that

$$
\sup _{B\left(x_{0}, r\right)} u \leqslant f_{S^{n-k}\left(x_{0}, r\right)} u(y) d H^{n-k}(y)+g(r)
$$

for $0<r<r_{0} / 2$. Here $k$ is an integer such that $1 \leqslant k<p$.

Proof. This follows immediately from Theorem 4.1 and (24), for setting $c(r)=$ $f_{S^{n-k}\left(x_{0}, r\right)} u(y) d H^{n-k}(y)$, we have

$$
\begin{aligned}
\mu(r)-c(r) & =f_{B\left(x_{0}, 7 r / 8\right)} \mu(r)-c(r) \\
& \leqslant f_{B\left(x_{0}, 7 r / 8\right)}|\mu(r)-u|+f_{B\left(x_{0}, 7 r / 8\right)}|u-c(r)| \\
& \leqslant C[\mu(r)-\mu(r / 2)+a(r)]^{p-1 / p} .
\end{aligned}
$$

Now set $g(r)=C[\mu(r)-\mu(r / 2)+a(r)]^{p-1 / p}$ and the conclusion follows.

5. Parabolic equations. In this section we consider parabolic equations of the form

$$
u_{t}=\operatorname{div} A\left(x, t, u, u_{x}\right)+B\left(x, t, u, u_{x}\right)
$$

where $A$ and $B$ are Baire functions defined on $\Omega_{T} \times R^{1} \times R^{n}$. Here $\Omega_{T}=\Omega \times(0, T)$ where $\Omega$ is an open subset of $R^{n}$.

The structure imposed on $A$ and $B$ is similar to that considered in the elliptic case, (12):

$$
\begin{gathered}
|A(x, t, u, w)| \leqslant a_{0}|w|+a_{1}|u|+a_{2}, \\
|B(x, t, u, w)| \leqslant b_{0}|w|^{2}+b_{1}|w|+b_{2}|u|+b_{3}, \\
A(x, t, u, w) \cdot w \geqslant|w|^{2}-c_{1}|u|^{2}-c_{2} .
\end{gathered}
$$

$a_{0}$ and $b_{0}$ are nonnegative constants and the remaining coefficients are required to lie in appropriate Lebesgue spaces (cf. [LSU, T2]), but as in $\$ 4$, for simplicity of exposition, we will assume that all coefficients are bounded by some constant $K>0$. A function $u \in W_{\text {loc }}^{1,2}\left(\Omega_{T}\right)$ is called a weak subsolution (supersolution) of (27) if

$$
\int-u \phi_{t}+A \cdot \nabla \phi-B \phi \leqslant 0 \quad(\geqslant 0)
$$

for all bounded $\phi \geqslant 0, \phi \in W_{0}^{1,2}\left(\Omega_{T}\right)$.

The object of this section is to establish results for subsolutions of (27) analogous to those in Theorems 4.3 and 4.6. For this purpose we consider an arbitrary point $Z_{0} \in \Omega_{T}$ and, for convenience, set $Z_{0}=(0,0)$. We shall utilize space-time cylinders of the following form:

$$
\begin{gathered}
R(r)=B(r) \times\left(\sigma_{1} r^{2}, \sigma_{2} r^{2}\right), \quad R^{-}(r)=B(\alpha r) \times\left(\tau_{1} r^{2}, \tau_{2} r^{2}\right), \\
R^{*}(r)=B(\beta r) \times\left(\rho_{1} r^{2}, \rho_{2} r^{2}\right),
\end{gathered}
$$


where $B(r)$ denotes the $n$-ball of radius $r$ and center $x_{0}=0$ and where

$$
0<\beta<\alpha<1, \quad \sigma_{1}<\rho_{1}<\rho_{2}<\rho_{3}<0<\rho_{4}<\sigma_{2} .
$$

Note that

$$
R\left(r / 2^{N}\right) \subset R^{-}(r)
$$

for some integer $N$. Finally, for $u \in W_{\mathrm{lox}}^{1.2}\left(\Omega_{T}\right)$ a subsolution of (27). let $\mu(r)=$ $\sup \{u(z): z \in R(r)\}$. The following result is proved in [T2] and its proof runs parallel to that of the elliptic version in Theorem 4.1 .

5.1. TheOREM. Let $u \in W_{\text {loc }}^{1.2}\left(\Omega_{T}\right)$ be a weak subsolution of (27) such that $0 \leqslant u(z)$ $\leqslant L$ for each $z \in \Omega_{T}$. There is a number $\gamma>1$ and a constant $C$ depending only on $L, n$, and the structures (28) and (30) such that if $R(r) \subset \Omega_{T}$. then

$$
\left(f_{R^{*}(r)}|\mu(r)-u|^{\gamma}\right)^{1 / \gamma} \leqslant C \min _{R^{-}(r)}[\mu(r)-u+a(r)]
$$

where $a(r)=r+K r$.

We now will establish an estimate on the growth of the $V^{2}$-norm of $\mu(r)-u$ where $u$ is a weak subsolution of (27). This estimate is similar to the one that appears in [Z2, Theorem 4.3] and is the parabolic analogue of the estimate that appears in Theorem 4.3 above.

For this purpose let $u$ be a bounded, nonnegative weak subsolution of (27) and let

$$
\phi=\eta^{2} u e^{h_{0} u},
$$

where $\eta$ is a smooth cut-off function that will be specified below. Because $u$ is assumed to be bounded, we may assume that the terms involving $a_{1}|u|, b_{2}|u|$, and $c_{1}|u|^{2}$ in (28) can be absorbed in $a_{2}, b_{3}$ and $c_{2}$, respectively. Therefore, substitution of (32) into (29) yields

$$
\begin{aligned}
\iint \eta^{2} e^{b_{0} u} u u_{t}+\iint \eta^{2} e^{b_{0} u}\left(1+b_{0} u\right) & \left(|\nabla u|^{2}-C_{2}\right) \\
\leqslant & \iint 2 \eta u e^{b_{0} u}\left[a_{0}|\nabla u|+a_{2}\right]|\nabla \eta| \\
& +\iint \eta^{2} e^{b_{0} u} u\left[b_{0}|\nabla u|^{2}+b_{1}|\nabla u|+b_{3}\right] .
\end{aligned}
$$

Now let $v=\mu(r)-u$ (for simplicity we will write $\mu=\mu(r))$ and define

$$
f(u)=b_{0}^{-1} e^{b_{0} u}\left(b_{0}^{-1}-u\right)-b_{0}^{-1} e^{b_{0} \mu}\left(b_{0}^{-1}-\mu\right) .
$$

Then

$$
\begin{gathered}
f^{\prime}(u)=-u e^{b_{0} u}, \quad f^{\prime \prime}(u)=-e^{b_{0} u}\left(1+b_{0} u\right), \\
\frac{1}{2}\left(\mu^{2}-u^{2}\right) \leqslant f(u) \leqslant \mu e^{b_{0} \mu}(\mu-u) \quad \text { for } 0 \leqslant u \leqslant \mu .
\end{gathered}
$$


There is a constant $C$ such that (33) can be written as

$$
\begin{aligned}
& \iint \eta^{2} \frac{\partial f(u)}{\partial t}+\iint \eta^{2}|\nabla u|^{2} \\
& \leqslant C\left[\iint \eta|\nabla u||\nabla \eta|+\iint \eta^{2}+\iint \eta|\nabla \eta|+\iint \eta^{2}|\nabla u|\right]
\end{aligned}
$$

The constant $C$ depends upon the structure (28) and the bound for $|u|$. Now define $R^{\prime}(r)=R^{*}(\gamma r)$ where $0<\gamma<1$, and choose $\eta$ so that $\eta \equiv 1$ on $R^{\prime}(r)$ and spt $\eta \subset$ $R^{*}(r)$. Refer to (30) and set $t_{1}=\rho_{1} r^{2}$ and $t_{2}=\rho_{2} r^{2}$. Choose $t^{*} \in\left(t_{1}, t_{2}\right)$ so that

$$
\int \eta^{2}\left(x, t^{*}\right) f^{2}\left(x, t^{*}\right) d x>\frac{1}{2} \sup _{\left(t_{1}, t_{2}\right)} \int \eta^{2}(x, t) f^{2}(x, t) d x .
$$

If we replace $t$ by $t^{*}$ in (36), we are led to the following estimate:

$$
\begin{aligned}
& \sup _{\left(t_{1}, t_{2}\right)} \int[\eta f(x, t)]^{2} d x+\iint \eta^{2}|\nabla v|^{2} d x d t \\
& \quad \leqslant C\left[\iint \eta|\nabla u||\nabla \eta|+\iint \eta^{2}+\iint \eta|\nabla \eta|+\iint \eta^{2}|\nabla u|+\iint \eta\left|\eta_{t}\right| f\right] .
\end{aligned}
$$

We now proceed exactly as in the proof of Theorem 4.3 to estimate the rate at which the right side of (37) tends to 0 . Indeed, by employing (31) and Theorem 5.1, we find that there is a constant $C$ depending only on the given data such that the first four terms on the right side of (37) are bounded by

$$
C r^{n}\left[\mu(r)-\mu\left(r / 2^{N}\right)+a(r)\right] .
$$

Refer to (35) to find that $|f(u)| \leqslant C_{1}|v|$ and, therefore, by using Theorem 5.1 again, we have

$$
\iint \eta\left|\eta_{t}\right||f(u)| \leqslant C r^{n}\left[\mu(r)-\mu\left(r / 2^{N}\right)+a(r)\right]
$$

Referring again to (35), the next lemma now follows from (37)-(39).

5.2. Lemma. Let $u \in W_{\text {loc }}^{1,2}\left(\Omega_{T}\right)$ be a bounded, nonnegative weak subsolution of (27). Then

$$
\sup _{\left(t_{1}, t_{2}\right)} \int_{B(\beta \gamma r)}[\mu(r)-u]^{2} d x+\iint_{R^{\prime}(r)}|\nabla u|^{2} d x d t \leqslant C r^{n}\left[\mu(r)-\mu\left(r / 2^{N}\right)+a(r)\right]
$$

whenever $R(r) \subset \Omega_{T}$.

We are now in a position to establish the parabolic analogue of Theorem 4.3. This result is concerned with the behavior of a weak subsolution in a neighborhood of an arbitrary point $z_{0} \in \Omega_{T}$. We will continue to assume that $z_{0}=(0,0)$ and the geometric configuration imposed by (30). 
5.3. ThEOREM. Let $u \in W_{\text {loc }}^{1,2}\left(\Omega_{T}\right)$ be a bounded, nonnegative weak subsolution of (27). For each $z_{0} \in \Omega_{T}$ with $R_{z_{0}}\left(r_{0}\right) \subset \Omega_{T}$ for some $r_{0}>0$, let $m_{t} \in M_{\alpha}^{+}, 1 \leqslant \alpha<2$, be a Radon measure supported on $\bar{B}\left(\beta \gamma r_{0}\right) \times\{t\}$. There is a constant $C$ depending only on the bound for $u$ and the given data such that

$$
f_{\gamma t_{1}}^{\gamma t_{2}} \int_{B(\beta \gamma r)}\left|u(x, t)-\int u \circ T_{r}(y, t) d m_{t}(y)\right|^{2} d x d t \leqslant C\left[\mu(r)-\mu\left(r / 2^{N}\right)+a(r)\right]
$$

for each $r \leqslant r_{0}$ where $T_{r}: B\left(\beta \gamma r_{0}\right) \times\{t\} \rightarrow B(\beta \gamma r) \times\{t\}$ is defined by $T_{r}(x, t)=$ $\left(r / r_{0} x, t\right)$.

Proof. Because $u \in W_{\text {loc }}^{1.2}\left(\Omega_{T}\right)$, it is an elementary fact concerning Sobolev functions that $u(\cdot, t) \in W_{\mathrm{loc}}^{1,2}(\Omega \times\{t\})$ for a.e. $t$. For all such $t$ apply Theorem 3.4 to obtain

$$
\int_{B(\beta \gamma r)}\left|u(x, t)-\int u \circ T_{r}(y, t) d m_{t}(y)\right|^{2} d x \leqslant C r^{2-n} \int_{B(\beta \gamma r)}|\nabla u(x, t)|^{2} d x .
$$

Now integrating with respect to $t$ from $\gamma t_{1}=\gamma \rho_{1} r^{2}$ to $\gamma t_{2}=\gamma \rho_{2} r^{2}$ and applying Lemma 5.2 yields the desired result.

5.4. Corollary. For each $z_{0} \in \Omega_{T}$ with $R_{z_{0}}\left(r_{0}\right) \subset \Omega_{T}$ for some $r_{0}>0$, there is a nonnegative function $g(r)$ with $\int_{0} g(r)^{2} d r / r<\infty$ such that

$$
\sup _{R(r)} u \leqslant f_{\gamma t_{1}}^{\gamma t_{2}} \int u \circ T_{r}(y, t) d m_{t}(y) d t+g(r)
$$

for $0<r \leqslant r_{0}$.

Proof. From Theorems 5.1 and 5.3 it follows that

$$
\begin{aligned}
& f_{\gamma t_{1}}^{\gamma t_{2}}\left[\mu(r)-\int u \circ T_{r}(y, t) d m_{t}(y)\right]^{2} d t \\
& \quad=f_{\gamma t_{1}}^{\gamma t_{2}} f_{B(\beta \gamma r)}\left[\mu(r)-\int u \circ T_{r}(y, t) d m_{t}(y)\right]^{2} d x d t \\
& \quad \leqslant C f_{R^{\prime}(r)}[\mu(r)-u]^{2}+C f_{\gamma t_{1}}^{\gamma t_{2}} \int_{B(\beta \gamma r)}\left|u(x, t)-\int u \circ T_{r}(y, t) d m_{t}(y)\right|^{2} d x d t \\
& \leqslant C\left[\mu(r)-\mu\left(r / 2^{N}\right)+a(r)\right] .
\end{aligned}
$$

Thus,

$$
f_{\gamma t_{1}}^{\gamma t_{2}}\left[\mu(r)-\int u \circ T_{r}(y, t) d m_{t}(y)\right] d t \leqslant C\left[\mu(r)-\mu\left(r / 2^{N}\right)+a(r)\right]^{1 / 2} .
$$

The result now follows if we set $g(r)=C\left[\mu(r)-\mu\left(r / 2^{N}\right)+a(r)\right]^{1 / 2}$.

As in $\S 4$, the most interesting case to consider is when the measure $m$ is taken as a geometric measure with certain homogeneity properties. For example, let

$$
m_{t}=H^{n-1} \mid S^{n-1}\left(x_{0}, \beta \gamma r_{0}\right) \times\{t\} \text {. }
$$


Then

$$
\int u \circ T_{r}(y, t) d m_{t}(y)=f_{S^{n-1}\left(x_{0}, \beta \gamma r\right) \times\{t\}} u(y, t) d H^{n-1}(y)
$$

and, therefore,

$$
\begin{aligned}
f_{\gamma t_{1}}^{\gamma t_{2}} \int u \circ T_{r}(y, t) d m_{t}(y) d t & =f_{\gamma t_{1}}^{\gamma t_{2}} f_{S^{n-1}\left(x_{0}, \beta \gamma r\right) \times\{t\}} u(y, t) d H^{n-1}(y) \\
& =f_{\partial^{*} R^{\prime}(r)} u(z) d H^{n}(z),
\end{aligned}
$$

where $\partial^{*} R^{\prime}(r)$ denotes the lateral boundary of the cylinder $R^{\prime}(r)$.

We have thus proved

5.5. Corollary. For each $z_{0} \in \Omega_{T}$ with $R_{z_{0}}\left(r_{0}\right) \subset \Omega_{T}$ for some $r_{0}>0$, there is a nonnegative function $g(r)$ with $\int_{0} g(r)^{2} d r / r<\infty$ such that

$$
\sup _{R(r)} u \leqslant \int_{\partial^{*} R^{\prime}(r)} u(z) d H^{n}(z)+g(r)
$$

for $0<r \leqslant r_{0}$.

As in $\$ \S 3$ and 4, one could establish similar results by making different selections for the measure $m$. For example, in addition to the choice made in the above corollary, one could also take $m_{t}=H^{n-1} \mid \Pi^{n-1}\left(x_{0}\right) \times\{t\}$, where $\Pi^{n-1}$ is an $(n-1)$-plane that is orthogonal to the $t$-axis.

\section{REFERENCES}

[AM] D. Adams and N. Meyers, Thinness and Wiener criteria for non-linear potentials, Indiana Univ. Math. J. 22 (1972), 169-197.

[F] H. Federer, Geometric measure theory, Springer-Verlag, New York, 1969.

[Fl] W. H. Fleming, Functions whose partial derivatives are measures, Illinois J. Math. 4 (1960), 452-478.

[FZ] H. Federer and W. P. Ziemer, The Lebesgue set of a function whose distributional derivatives are $p$-th power summable, Indiana Univ. Math. J. 22 (1972), 139-158.

[GT] D. Gilbarg and N. Trudinger, Elliptic partial differential equations of second order, Springer-Verlag, New York, 1977.

[GZ] R. Gariepy and W. P. Ziemer, A regularity condition at the boundary for solutions of quasilinear elliptic equations, Arch. Rational Mech. Anal. 67 (1977), 25-39.

[LU] O. A. Ladyzhenskaya and N. N. Uraltseva, Linear and quasilinear elliptic equations, Academic Press, New York, 1968.

[M] N. Meyers, Integral inequalities of Poincaré and Wirtinger type, Arch. Rational Mech. Anal. 68 (1978), 113-120.

[Mo] J. Moser, A Harnack inequality for parabolic differential equations, Comm. Pure Appl. Math. 17 (1964), 101-134.

[MZ] N. Meyers and W. P. Ziemer, Integral inequalities of Poincaré and Wirtinger type for BV functions, Amer. J. Math. 99 (1977), 1345-1360.

[S] J. Serrin, Local behavior of solutions of quasilinear elliptic equations, Acta Math. 111 (1964), 247-302.

[T1] N. Trudinger, On Harnack type inequalities and their application to quasilinear elliptic equations, Comm. Pure Appl. Math. 20 (1967), 721-747.

[T2] _ Pointwise estimates and quasilinear parabolic equations, Comm. Pure Appl. Math. 21 (1968), 205-266.

[Z1] W. P. Ziemer, Interior and boundary continuity of weak solutions of degenerate parabolic equations, Trans. Amer. Math. Soc. 271 (1982), 733-748.

[Z2] _ Behavior at the boundary of solutions of quasilinear parabolic equations, J. Differential Equations 35 (1980), 291-305.

DEPARTMENT OF MATHEMATICS, INDIANA UNIVERSity, Bloomington, INDIANA 47401 